\section{Epithelial-mesenchymal transition: potential role in obliterative bronchiolitis?}

\author{
Brigham C Willis, ${ }^{1,2}$ Zea Borok ${ }^{3}$
}

Lung transplantation remains the only viable option for many patients suffering from a variety of progressive or intractable end-stage lung diseases. Despite significant advances in the prevention of early graft rejection, ischaemia-reperfusion injury and acute management of lung transplant recipients, significant challenges remain in the chronic management of patients after lung transplantation. ${ }^{1}$ In this issue of Thorax, Borthwick and colleagues $^{2}$ provide intriguing new evidence that implicates the airway epithelium directly in the pathogenesis of bronchiolitis obliterans syndrome (BOS), the most significant factor in determining long-term lung graft survival (see page 770). As discussed in the study, the pathological lesion of BOS is obliterative bronchiolitis $(O B)$, which recently has been postulated to be at least partially a disease of aberrant epithelial repair processes. ${ }^{3}$ Borthwick and colleagues provide evidence that epithelial to mesenchymal transition (EMT), a process whereby epithelial cells undergo a complete lineage transition to become fibroblasts and/or myofibroblasts, may underlie the dysfunctional airway repair processes that lead to $O B$. This study, and others like it, attempt to redefine traditional paradigms regarding normal airway epithelial biology and disease pathogenesis, and have the potential to lead to entirely new therapeutic avenues for previously untreatable disease processes such as BOS.

$\mathrm{OB}$ is characterised by initial inflammation of the small airways followed by airway remodelling, aberrant epithelial regeneration and repair, proliferation of fibroblasts and myofibroblasts, deposition

\footnotetext{
${ }^{1}$ Heart and Lung Institute, St Joseph's Hospital and Medical Center, Phoenix, Arizona, USA; ${ }^{2}$ Department of Pediatrics, University of Arizona, Tucson, Arizona, USA; ${ }^{3}$ Division of Pulmonary and Critical Care Medicine, Will Rogers Institute Pulmonary Research Center, Keck School of Medicine, University of Southern California, Los Angeles, California, USA
}

Correspondence to: Dr B C Willis, Heart and Lung Institute, 500 Thomas Rd, Suite 500, St Joseph's Hospital and Medical Center, Phoenix, AZ 85260, USA; brigham.willis@chw.edu of extracellular matrix (ECM) and eventual airway obstruction. ${ }^{4}$ The initial inflammatory response is the result of an allogeneic immune response initiated against donor antigens in the graft endothelial and airway epithelial cells. This response characteristically generates antigen-specific graft-infiltrating destructive lymphocytes. The lymphocytes facilitate activation of macrophages and a variety of other inflammatory cells, with resultant epithelial damage. ${ }^{5}$ The critical role of this allogeneic immune response as the initial trigger leading to $O B$ is supported by the fact that the primary risk factors for the development of BOS after lung transplantation are class I and II mismatches between donor and recipient, as well as the number and severity of rejection episodes. ${ }^{4}$ Although it has been recognised for over a decade that the airway epithelium is a target of the initial immune response, ${ }^{6}$ the pathogenetic pathway that leads to disruption of normal epithelial repair processes, excessive fibroblastic responses and resultant excessive ECM deposition and eventual obliteration of small airways is still incompletely elucidated.

While bronchial epithelial cells (BECs) have been shown to directly present antigen $^{7}$ and are potentially the primary target of immunological attack during the pathogenesis of $\mathrm{OB},{ }^{8}$ their precise link to the proliferation of fibroblasts and the propagation of fibrosis is not entirely clear. It is known that epithelial cell apoptosis and disruption of epithelial integrity probably contributes to subepithelial fibroblastic proliferation, ${ }^{9}$ but the primary source of the proliferating fibroblasts during airway fibrosis is still unknown. One possible source is direct conversion of proliferating BECs into pathogenetic fibroblasts and myofibroblasts through EMT.

EMT is a process by which epithelial cells lose fundamental epithelial characteristics such as tight junctions, apical: basolateral polarity and the expression of epithelial-specific markers and assume a mesenchymal phenotype, expressing a variety of mesenchymal markers and acquiring functional characteristics of fibroblasts and myofibroblasts such as ECM production, motility and the ability to invade surrounding tissues. ${ }^{10}$ EMT is not a new concept, being critical during normal development and for the development of metastatic potential and increased invasiveness during cancer progression. ${ }^{11}$ However, the role of EMT in the pathogenesis of adult tissue fibrosis is a relatively new area of study. EMT has been most commonly investigated as a mechanism underlying fibrosis in renal and lens epithelium. In the kidney, approximately $40 \%$ of new fibroblasts are thought to arise via EMT during injury ${ }^{12}$ while, in the eye, EMT has been demonstrated both in vitro and in vivo. ${ }^{13}$ Very recently, the role of EMT in the pathogenesis of pulmonary disease has begun to be investigated. The first demonstrations of EMT in pulmonary epithelium were in alveolar epithelial cells. In response to transforming growth factor $\beta$ (TGF $\beta$ ) in vitro, several groups have demonstrated conversion of alveolar epithelial cells to a myofibroblast-like phenotype, with a complete morphological and functional change and acquisition of mesenchymal markers concomitant with the loss of detectable epithelial characteristics. ${ }^{14-16}$ Furthermore, EMT has been demonstrated in alveolar epithelium in vivo in mice. In one recent study, lineage-tagged alveolar epithelial cells were shown to contribute over $30 \%$ of the alveolar fibroblastic response during experimental lung injury. ${ }^{17}$ Finally, immunohistochemical evidence from lung biopsy samples from patients with idiopathic pulmonary fibrosis (IPF) suggests that up to $80 \%$ of the epithelium in areas of active fibrosis exhibit evidence of EMT. ${ }^{14}$ Given the growing body of evidence implicating EMT of alveolar epithelial cells in the pathogenesis of pulmonary fibrosis, the possibility that BECs may undergo EMT to contribute to airway remodelling and fibrosis is intriguing.

In this issue, Borthwick et al cultured primary human BECs in the presence and absence of TGF $\beta$ and tumour necrosis factor $\alpha(\mathrm{TNF} \alpha)$. Only TGF $\beta$ alone was able to induce a fibroblastoid morphology, but the combination of both TGF $\beta$ and TNF $\alpha$ resulted in the most robust phenotypic change together with upregulation of mesenchymal markers (vimentin and fibronectin) and downregulation of the epithelial markers cytokeratin 19 and Ecadherin. Treated cells also acquired functional characteristics of myofibroblasts, 
demonstrating an increase in the ability to invade collagen and deposit ECM. The authors extended their study to examine normal lung and pathological specimens from stable transplant patients and those with progressive BOS. They used quantitative immunohistochemistry to demonstrate a correlation between disease severity and both loss of epithelial markers and acquisition of mesenchymal markers in the bronchial epithelium. Finally, they used confocal microscopy to demonstrate co-localisation of epithelial and mesenchymal markers within the epithelium of transplant recipients with $\mathrm{OB}$. Together, the findings of this study add support to the hypothesis that EMT may play a significant role in the pathogenesis of $\mathrm{OB}$.

While few, there have been other suggestions of EMT as a potential pathogenetic mechanism in $\mathrm{OB}$. An early report that included authors of the current study reported that $15 \%$ of the epithelium in biopsy specimens from clinically stable transplant patients co-stained for the fibroblast marker S100A4. ${ }^{3}$ Recently, mouse tracheal epithelial cells were shown to undergo at least partial EMT in response to TGF $\beta_{1}$, possibly through a JNK-1 mediated mechanism. ${ }^{18}$ Finally, peribronchial fibrosis during bleomycininduced lung injury was found to contain a subset of BECs possibly derived from EMT. ${ }^{19}$ Clearly, the possibility that BECs can contribute to the airway fibrotic response in a variety of disease processes deserves further investigation.

An intriguing aspect of the current study is the requirement for both TGF $\beta$ and TNF $\alpha$ together to induce a robust EMT response in BECs in vitro. A potential criticism of previous studies has been that the dose of TGF $\beta$ required to induce EMT in BECs has been higher than that required to induce alveolar EMT (5-50 ng/ml vs $0.5-1 \mathrm{ng} / \mathrm{ml}) .{ }^{18}$ Given that physiological concentrations of TGF $\beta_{1}$ during lung injury only reach concentrations of approximately $1 \mathrm{ng} / \mathrm{ml}$ or less, ${ }^{20}$ the possibility that bronchial EMT is a purely in vitro phenomenon has been raised. The combination of TGF $\beta$ and TNF $\alpha$ is intriguing in that EMT during airway fibrosis would probably occur in the presence of both of these cytokines due to the ongoing inflammation during rejection. The addition of $\mathrm{TNF} \alpha$ resulted in the induction of EMT at doses of TGF $\beta$ as low as $1 \mathrm{ng} / \mathrm{ml}$, within the physiological range. This synergy between TGF $\beta$ and TNF $\alpha$ confirms earlier observations in EMT of alveolar epithelial cells, ${ }^{14}$ and suggests an interesting cross-talk between TGF $\beta$ and TNF $\alpha$-induced intracellular signalling pathways.

In summary, Borthwick and colleagues ${ }^{2}$ have provided additional evidence for the possibility of EMT as a pathogenetic contributor to airways fibrosis in $\mathrm{OB}$ and perhaps other diseases of the small airways. Studies like this force us to reexamine long-held disease paradigms and hopefully embark on new investigations to find novel therapeutics. Given the historical lack of progress in the prevention and treatment of BOS, new insights and avenues of inquiry are critical to improving the outcomes and lives of patients after lung transplantation.

Funding: This work was supported by the St Joseph's Hospital Foundation, the Hastings Foundation and by grant R01 HL089445 from the National Institutes of Health to ZB. ZB holds the Ralph Edgington Chair in Medicine.

Competing interests: None.

Provenance and peer review: Commissioned; not externally peer reviewed.

Thorax 2009;64:742-743. doi:10.1136/thx.2009.114413

\section{REFERENCES}

1. Wilkes DS, Egan TM, Reynolds HY. Lung transplantation: opportunities for research and clinica advancement. Am J Respir Crit Care Med 2005;172:944-55.

2. Borthwick LA, Parker SM, Brougham KA, et al. Epithelial to mesenchymal transition (EMT) and airway remodelling after human lung transplantation. Thorax 2009:64:770-7.

3. Ward C, Forrest IA, Murphy DM, et al. Phenotype of airway epithelial cells suggests epithelial to mesenchymal cell transition in clinically stable lung transplant recipients. Thorax 2005;60:865-71.
4. Jaramillo AF, Fernandez G, Kuo EY, et al. Immune mechanisms in the pathogenesis of bronchiolitis obliterans syndrome after lung transplantation. Pediatr Transplant 2005:9:84-93.

5. Paradis I. Bronchiolitis obliterans: pathogenesis, prevention, and management. Am J Med Sci 1998;315:161-78.

6. Snell GI, Ward C, Wilson JW, et al. Immunopathological changes in the airways of stable lung transplant recipients. Thorax 1997;52 322-8.

7. Reznik SI, Jaramillo A, Zhang L, et al. Anti-HLA antibody binding to HLA class I molecules induces proliferation of airway epithelial cells: a potential mechanism for bronchiolitis obliterans syndrome. J Thorac Cardiovasc Surg 2000;119:39-45.

8. Plissonnier D, Henaff $M$, Poncet $P$, et al. Involvement of antibody-dependent apoptosis in graft rejection. Transplantation 2000;69:2601-8.

9. Morishima $\mathbf{Y}$, Nomura A, Uchida $Y$, et al. Triggering the induction of myofibroblast and fibrogenesis by airway epithelial shedding. Am J Respir Cell Mol Biol 2001;24:1-11.

10. Kalluri R, Neilson EG. Epithelial-mesenchymal transition and its implications for fibrosis. J Clin Invest 2003;112:1776-84.

11. Slack JM, Tosh D. Transdifferentiation and metaplasia—switching cell types. Curr Opin Genet Dev 2001;11:581-6.

12. Iwano M, Plieth D, Danoff TM, et al. Evidence that fibroblasts derive from epithelium during tissue fibrosis. J Clin Invest 2002;110:341-50.

13. Saika S, Kono-Saika S, Ohnishi Y, et al. Smad3 signaling is required for epithelial-mesenchymal transition of lens epithelium after injury. Am J Pathol 2004;164:651-63.

14. Willis BC, Liebler JM, Luby-Phelps K, et al. Induction of epithelial-mesenchymal transition in alveolar epithelial cells by transforming growth factor- $\beta 1$ : potential role in idiopathic pulmonary fibrosis. Am J Pathol 2005;166:1321-32.

15. Yao HW, Xie OM, Chen JQ, et al. TGF- $\beta 1$ induces alveolar epithelial to mesenchymal transition in vitro. Life Sci 2004;76:29-37.

16. Kasai H, Allen JT, Mason RM, et al. TGF- $\beta 1$ induces human alveolar epithelial to mesenchymal cell transition (EMT). Respir Res 2005;6:56

17. Kim KK, Kugler MC, Wolters PJ, et al. Alveolar epithelial cell mesenchymal transition develops in vivo during pulmonary fibrosis and is regulated by the extracellular matrix. Proc Natl Acad Sci U S A 2006;103:13180-5.

18. Alcorn JF, Guala AS, van der Velden J, et al. Jun Nterminal kinase 1 regulates epithelial-to-mesenchymal transition induced by TGF- $\beta 1$. J Cell Sci 2008;121(Pt 7):1036-45

19. Wu Z, Yang L, Cai L, et al. Detection of epithelial to mesenchymal transition in airways of a bleomycin induced pulmonary fibrosis model derived from an alpha-smooth muscle actin-Cre transgenic mouse. Respir Res 2007;8:1.

20. Hagimoto N, Kuwano K, Inoshima I, et al. TGF- $\beta 1$ as an enhancer of fas-mediated apoptosis of lung epithelial cells. J Immunol 2002;168:6470-8. 